Non-woven textile materials with antimicrobial properties are of high demands for applications ranging from medical dressing to everyday cleaning products. We proposed a plasma assisted route to engineer polymer films and nonwoven fabrics with antimicrobial nano-composite coatings. The project aims to optimize atmospheric pressure plasma deposition in order to engineer the coatings with high antibacterial activity and low cytotoxicity. Different methods of deposition are tested including radio-frequency plasma, direct current discharge in nitrogen as well barrier discharge in air with two methods of nano-particles incorporation: aerosol and impregnation. Nano-particles $\mathrm{Ag}$, $\mathrm{Cu}$ and $\mathrm{ZnO}$ are tested as antimicrobial agents with average nano-particle size of about $50 \mathrm{~nm}$. Nanoparticles are incorporated in between two layers of an organosilicon film with an impregnation process or alternatively through an aerosol injection step with averaged concentration of the nano-particles in the solution ranging in between 10 to $1000 \mathrm{mg} / \mathrm{l}$. Incorporation is made in a "sandwich-like structure" where a top layer coating (barrier) of 5 - $50 \mathrm{~nm}$ thickness is used for precise control of metal ions release and a reservation layer of about $200 \mathrm{~nm}$ is used for NPs load. The effect of the barrier coating on the surface concentration of nano-particles is determined by XPS. It is shown that an increase of the thickness of the barrier layer from 5 to $25 \mathrm{~nm}$ leads to a decrease of $\mathrm{Ag}$ content from 0.24 to 0.08 at. \%. Similar effect is found for nano-particles of $\mathrm{Cu}$ and $\mathrm{ZnO}$. Antibacterial efficiency of the samples against $P$. aeruginosa ATCC 9027 and $S$. aureus $M u 50$ bacteria shows that all treated samples exhibit higher antibacterial efficiency against $S$. aureus. The antibacterial efficiency of AgNPs and CuNPs is above $90 \%$ which is practically interesting for medical application while ZnONPs shows lower antibacterial efficiency. Cytotoxicity of the coatings and materials is investigated for different deposition conditions and analyzed in terms of possible medical applications.

This work is supported by the M.Era-Net project IWT 140812 "PlasmaTex".

\title{
Authors
}

\section{Anton Nikiforov}

Ghent University

Christophe Leys

Ghent University

Iryna Kuchakova

Ghent University

Myriam Vanneste

CentexBel

Pieter Heyse

CentexBel

Mike De Vrieze

CentexBel
Andrea Zille

University of Minho

Gheorghe Dinescu

NILPRP

Bogdana Mitu

NILPRP

Martina Modic

Jozef Stefan Institute

Uros Cvelbar

Jozef Stefan Institute

\section{View Related Events}

Day: Tuesday, 30 May 2017 
